\title{
Ist mehr Bilanzkontrolle eigentlich immer besser?
}

\author{
Alfred Wagenhofer*
}

Der Fall Wirecard schlug hohe Wellen, und das nicht nur in Deutschland. Dass bei einem DAX-Unternehmen rund zwei Milliarden Euro Vermögen offenbar erfunden waren und dieses nach der Aufdeckung dessen insolvent wurde, erschütterte das Vertrauen in die mit der Kontrolle von Jahresabschlüssen befassten Institutionen erheblich.

Die klassische Reaktion der Politik auf Bilanzskandale ist eine Verschärfung der gesetzlichen Regelungen. Dies lässt sich über 200 Jahre nachverfolgen (siehe Hail et al. 2018). Eine solche Verschärfung erfolgte kürzlich auch wieder mit dem im Juni 2021 beschlossenen Finanzmarktintegritätsstärkungsgesetz (FISG), das die Bilanzkontrolle grundlegend reformiert, indem es der Bundesanstalt für Finanzdienstleistungsaufsicht (BaFin) mehr Kompetenzen einräumt, das neue Regeln für die Abschlussprüfung schafft und Haftungen erhöht.

Die letzten ähnlich spektakulären Fälle traten Anfang der 2000er Jahre auf. In den USA gelangte Enron im Jahr 2001 zu Berühmtheit, weil es neben Standardmethoden, wie einer falschen Erfassung von Erträgen, auch innovative Methoden nutzte, wie z.B. Special Purpose Entities, womit Verbindlichkeiten aus dem Konzernabschluss herausgehalten werden konnten. In den USA war die regulative Reaktion der Sarbanes-Oxley Act 2002, der u.a. Interne Kontrollsysteme und Abschlussprüferaufsicht verstärkte. Auch in Deutschland gab es zu dieser Zeit spektakuläre Fälle, wenngleich in kleinerem Umfang, wie z.B. ComROAD, bei dem ein Großteil des Umsatzes offenbar auf Scheinrechnungen beruhte. Als Reaktion wurde u.a. das zweistufige Enforcementverfahren mit dem Bilanzkontrollgesetz 2004 eingeführt, das nun reformiert wird.

Diesen Maßnahmen zugrunde liegt die Erwartung, dass eine Verstärkung der Bilanzkontrolle die Qualität der Rechnungslegung - und damit das Vertrauen in den Finanzmarkt - erhöht. Gemäß dem Regierungsentwurf zum FISG (Februar 2021) gibt es in der Begründung des Gesetzes dazu keine Alternativen. Eine ähnliche Diskussion läuft in Großbritannien nach

Prof. Dr. Dr. h.c. Alfred Wagenhofer | Institut für Unternehmensrechnung und Controlling | Center for Accounting Research | Karl-Franzens-Universität Graz |

alfred.wagenhofer@uni-graz.at 
dem Carillion-Fall, wo das Financial Reporting Council durch einen neuen unabhängigen Regulator ersetzt werden soll.

Allerdings muss man auch zur Kenntnis nehmen, dass vorgenommene Verschärfungen später einzelne Skandale nicht verhindern konnten. Viel relevanter wäre es zu wissen, wie effektiv und effizient das System der Bilanzkontrolle insgesamt ist - und sein soll. Das Problem dabei besteht darin, dass nicht beobachtbar ist, was passiert wäre, hätte man eine Verschärfung von Regeln nicht vorgenommen. Hat dies Manipulationen sehr wohl verringert, und ist ein dennoch auftretender Bilanzskandal „nur" ein Ausreißer? Oder hätten die neuen Regeln retrospektiv betrachtet den jeweiligen Skandal verhindern können? Es gibt also nur eine einzige Geschichte und nicht mehrere parallele Geschichten.

Im Folgenden möchte ich einige Ergebnisse aus der spieltheoretischen Forschung vorstellen. Theoretische Forschung hat gegenüber empirischer Forschung den Vorteil, dass sie gezielt kausale Zusammenhänge untersucht. Damit kann die Frage, wie eine bestimmte Verschärfung der Bilanzkontrolle auf Manipulation und deren Folgen wirkt, unmittelbar beantwortet werden. Der Nachteil liegt allerdings darin, dass die Modelle zum Teil starke Vereinfachungen gegenüber der Realität erfordern, die ihre Verallgemeinerung schwierig erscheinen lassen. Es lassen sich aber zumindest mögliche nicht intendierte Folgen erkennen. Insofern ergänzen sich verschiedene Forschungsmethoden für die Generierung von Wissen.

\section{Kontrollmechanismen für die Finanzberichterstattung}

Die Finanzberichterstattung der Unternehmen von öffentlichem Interesse (vor allem kapitalmarktorientierte Unternehmen) ist stark reguliert. Es gibt umfangreiche Rechnungslegungsvorschriften (IFRS und HGB), die aufgrund der steigenden Komplexität der Geschäftsfälle ebenfalls umfangreicher und komplizierter werden. Deren jährliche Finanzberichterstattung umfasst den Jahresabschluss, Lagebericht sowie weitere Berichte, wie insbesondere die nichtfinanzielle Erklärung. 


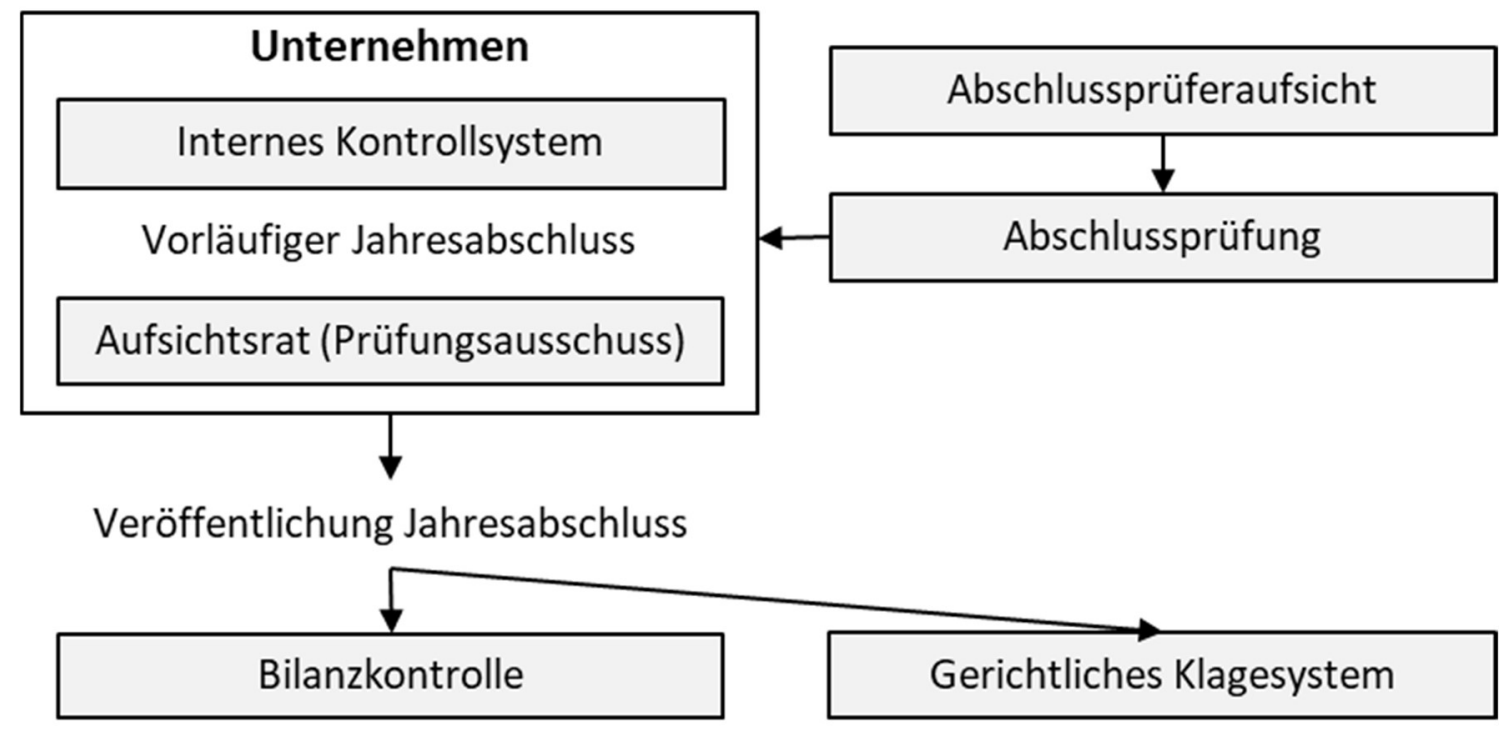

Abb. 1: Kontrollumfeld von Jahresabschlüssen.

Abbildung 1 gibt die wichtigsten Mechanismen wieder, die sich mit der Kontrolle der Finanzberichterstattung (im Folgenden mit Fokus auf den Jahresabschluss) befassen. Diese bestehen aus unternehmensinternen Mechanismen und externen Kontrollinstanzen. Unternehmen haben ein internes Kontroll- und des Risikomanagementsystems im Hinblick auf den Rechnungslegungsprozess einzurichten. Der erstellte Jahresabschluss ist von einem externen Abschlussprüfer zu prüfen, der selbst der Aufsicht durch eine Abschlussprüferaufsicht unterliegt. Der Aufsichtsrat überwacht die Geschäftsführung und richtet (nach FISG künftig zwingend) einen Prüfungsausschuss ein, der den Rechnungslegungsprozess, das interne Kontrollsystem und die Abschlussprüfung überwacht. Danach wird der Jahresabschluss veröffentlicht. Die bisher genannten Mechanismen versuchen Fehler und Manipulationen bereits vor deren Entstehen zu verhindern oder zu korrigieren. Deshalb ist ihre Effektivität von außen nicht ersichtlich.

Nach der Veröffentlichung unterliegt der Jahresabschluss der Bilanzkontrolle, wie der BaFin und der DPR. Neben der Bilanzkontrolle besteht für Investoren die Möglichkeit, den Gerichtsweg einzuschlagen und Schadenersatz einzuklagen, sofern Indizien für Manipulation bekannt werden. In diesen Fällen werden entdeckte Fehler allgemein bekannt.

Einige dieser Kontrollmechanismen existieren schon sehr lange, selbst wenn auch ihre Aufgaben zum Teil ausgeweitet wurden. Andere sind erst in jüngerer Zeit dazugekommen. Und es gibt weitere Kontrollmechanismen neben den oben genannten, wie z.B. Interne Revision, eine zweistufige Bilanzkontrolle (wie DPR und BaFin), Joint Audits usw. Komplizierend kommt hinzu, dass sich die Aufgaben zum Teil überschneiden, dass es i.d.R. keine Koordination der einzelnen Mechanismen und gegenseitigen Informationsaustausch gibt. Daher ist das Zusammenwirken dieser Kontrollmechanismen komplex. 


\section{Systematische Analyse}

Im Folgenden betrachten wir deshalb vereinfachende Situationen, um Wirkungen der Verstärkung einer Bilanzkontrolle aufzuzeigen, die in komplexeren Situationen ebenfalls auftreten. Wir gehen dabei nicht auf direkte Kosten der Bilanzkontrolle ein. Berücksichtigt man solche, reduziert dies mögliche Vorteile der Bilanzkontrolle.

\subsection{Abschlussprüfung und nichtstrategische Bilanzkontrolle}

Abb. 2 zeigt die erste Fallkonstellation, bestehend aus dem Unternehmen (repräsentiert durch den Vorstand), aus der Abschlussprüfung und der Bilanzkontrolle. Angenommen, das Unternehmen hat einen Anreiz, ein hohes Jahresergebnis auszuweisen, z.B. durch extensive Nutzung von Ermessensspielräumen bis hin zu falscher Bilanzierung (der Kürze wegen nennen wir dies „Manipulation“). Manipulation ist mit Wahrscheinlichkeit $m$ erfolgreich, wobei $m=0$ keine Manipulation bedeutet, und mehr Anstrengung zu Manipulation verursacht überproportional steigende Kosten. Die durchgezogenen Pfeile in Abb. 2 symbolisieren die zeitliche Abfolge der Entscheidungen, die strichlierten Pfeile die indirekte Entscheidungsbeeinflussung durch nachfolgende Kontrollinstanzen.

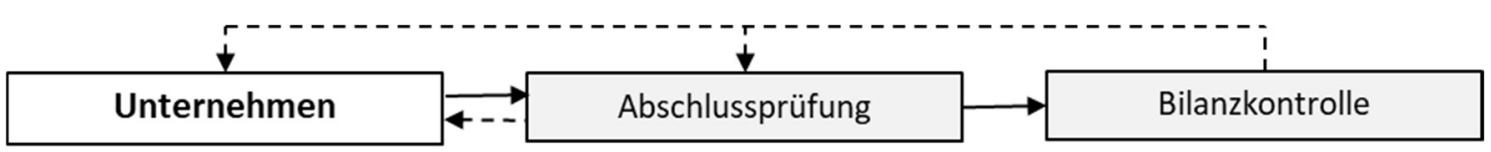

Abb. 2: Abschlussprüfung und Bilanzkontrolle.

Der Abschlussprüfer findet Manipulation mit Wahrscheinlichkeit $a$ und korrigiert den Jahresabschluss entsprechend. Eine Prüfungsintensität in Höhe von a verursacht überproportional steigende Prüfungskosten. Die Bilanzkontrolle prüft das Unternehmen stichprobenartig und findet im Fall einer Untersuchung (zusammen eine Wahrscheinlichkeit von $b$ ) eine verbliebene Manipulation, was öffentlich kommuniziert wird.

\subsubsection{Intendierte Wirkungen}

Insgesamt ergibt sich damit Folgendes: Die ursprüngliche Fehlerwahrscheinlichkeit ist $m$, nach der Abschlussprüfung sinkt sie auf $m(1-a)$, und nach der Bilanzkontrolle sinkt sie weiter auf $m(1-a)(1-b)$. Eine Verstärkung der Bilanzkontrolle erhöht $b$, und dies reduziert die Fehlerwahrscheinlichkeit entsprechend der obigen Formel. Ein strategisch agierendes Unternehmen antizipiert, dass Manipulation aufgrund eines höheren $b$ mit geringerer Wahrscheinlichkeit erfolgreich ist, und reduziert das Ausmaß der Manipulation gegenüber früher, weil dessen Nutzen gegenüber den Kosten sinkt. Dies ist die intendierte Wirkung. 
Überdies hat ein von der Bilanzkontrolle aufgedeckter Fehler negative Folgen für Unternehmen und Abschlussprüfer, die sowohl monetär als auch nichtmonetär, z.B. durch Reputationseffekte, sein können. Dadurch reduzieren sich die Manipulationsanreize weiter.

Unmittelbar ersichtlich ist aber auch, dass durch das Sinken von $m$ auch die Wahrscheinlichkeit sinkt, dass die Bilanzkontrolle Fehler aufdeckt - es gibt einfach weniger Manipulation. Manchmal wird die Fehleraufdeckungsrate als Performancemaß einer Bilanzkontrolle herangezogen, und im Lichte des obigen Zusammenhangs würde dies zu einer falschen Beurteilung der Effektivität der Bilanzkontrolle führen. ${ }^{1}$

\subsubsection{Weitere Wirkungen}

Es gibt aber noch mehr zu bedenken: Auch Abschlussprüfer sind strategisch agierende Institutionen, die ihren Prüfungseinsatz abhängig vom Klientenrisiko beurteilen. Sinkt aufgrund einer effektiveren Bilanzkontrolle das Manipulationsrisiko $m$, so können sie vielleicht die Prüfungsintensität herunterfahren. Dies erhöht umgekehrt das Manipulationsrisiko wieder etwas. Faktisch hängt also der Umfang an Manipulation von der Prüfungsintensität und der Kontrolleffektivität ab, d.h., $m=m(\hat{a}, b)$, wobei das „Dach“ die Erwartung des Unternehmens über a symbolisiert, weil $a$ im Gegensatz zu $b$ nicht bekannt oder beobachtbar ist. Gleichzeitig ist die Prüfungsintensität abhängig vom geschätzten Manipulationsrisiko und der Kontrolleffektivität, d.h., $a=a(\hat{m}, b)$.

Ewert und Wagenhofer (2019) analysieren eine solche Situation. Das spieltheoretische Lösungskonzept ist ein Gleichgewicht in rationalen Erwartungen, in dem Unternehmen und Abschlussprüfer ihren jeweiligen Erwartungsnutzen maximieren und die Erwartungen den tatsächlichen Strategien entsprechen, d.h., es gilt $\hat{m}=m$ und $\hat{a}=a$. Ewert und Wagenhofer (2019) zeigen Folgendes:

- Manipulation sinkt mit steigender Effektivität der Bilanzkontrolle (b) eindeutig. Dies entspricht dem intendierten Zweck.

- Die Prüfungsintensität steigt mit einer Erhöhung von $b$ zunächst, sinkt aber dann wieder. D.h., eine effektive Bilanzkontrolle kann die Prüfungsintensität substituieren, wodurch die Prüfungsqualität sinkt.

- Hält man sich vor Augen, dass eine Abschlussprüfung i.d.R. signifikant umfangreicher ist als eine ex post Bilanzkontrolle, welche oft nur vom Schreibtisch aus erfolgt, führt eine Substitution von $a$ durch $b$ zu einer insgesamt geringeren Qualität der Finanzberichterstattung. Dies ist ein nicht intendierter Effekt.

\footnotetext{
Man stelle sich nur vor, was passiert, wenn die Bilanzkontrolle sämtliche Fehler aufdecken könnte. In diesem Fall verhindert sie Manipulation vollständig, mit dem Ergebnis, dass sie dann keine Fehler mehr findet. Wiederum ist die Unbeobachtbarkeit von ursprünglich vorgenommener Manipulation schuld, dass die Fehleraufdeckungsrate alleine nicht aussagekräftig ist.
} 


\subsection{Strategische Bilanzkontrolle und Investorenklagen}

Der bisherigen Analyse lag die Annahme zugrunde, dass die Bilanzkontrolle kein strategischer „Spieler“ ist, sondern ihre Kontrolle mit allgemein bekannter Effektivität plangemäß durchführt. Aber auch die Bilanzkontrolle wird von Menschen durchgeführt, die Anreize haben. Ein solcher Anreiz kann sein, möglichst zu vermeiden, dass hinterher ein Fehler öffentlich bekannt wird, der in der Kontrolle nicht erkannt wurde, und der Bilanzkontrolle also öffentlich Versagen vorgeworfen werden könnte. Dies ist mit direkten Kosten oder Reputationskosten verbunden. Man betrachte diesbezüglich nur die Auswirkungen von Wirecard auf deren Präsidenten.

Schantl und Wagenhofer (2020) analysieren eine Situation mit einer strategisch agierenden Bilanzkontrolle. Die Spieler sind das Unternehmen, die Bilanzkontrolle und als zweiter Kontrollmechanismus gerichtliche Klagen von Investoren als eine Form privater Rechtsdurchsetzung. Die Bilanzkontrolle nimmt Stichprobenprüfungen vor, deren Umfang bekannt ist, und wählt strategisch die nicht beobachtbare Kontrollintensität $c$. Die Fehleraufdeckungsrate ergibt sich dann als Produkt von Stichprobenumfang und Kontrollintensität. Ein höheres $c$ verursacht überproportional steigende Kosten oder Disnutzen der dort Tätigen.

Die Investoren beobachten, wenn die Bilanzkontrolle einen Fehler findet, weil dies öffentlich bekanntgemacht wird. Dann können sie unmittelbar das Unternehmen vor Gericht wegen Manipulation klagen. Andernfalls wissen Sie jedoch nicht, ob das Unternehmen nicht in der Stichprobe war oder ob eine Kontrolle stattfand und kein Fehler entdeckt wurde. In der Folge beobachten die Investoren ein Signal, wie ein späteres schlechtes Jahres- oder Zwischenergebnis, ein negativer Medienbericht oder Ähnliches. Wenn dieses Signal auf Manipulation des Jahresergebnisses hindeutet, können sie das Unternehmen ebenfalls verklagen. Konkret klagen sie für ungünstige Signale, und die Grenze, unter welcher geklagt wird, sei mit $k$ bezeichnet. Den Investoren entstehen Klagekosten, und je niedriger diese sind, desto eher werden sie klagen, was einem systematisch hohen „Klagerisiko“ gleichkommt. Im Gerichtsverfahren kommt heraus, ob Manipulation stattgefunden hat; in diesem Fall wird gegen das Unternehmen eine Strafe verhängt. Gleichzeitig kommt heraus, ob die Bilanzkontrolle tätig war oder nicht. Hat sie kontrolliert, entstehen ihr ebenfalls Kosten, weil sie ihre Aufgabe offenbar „nicht ordentlich“ gemacht hat.

Abb. 3 gibt diese Situation wieder. Wie schon in Abb. 2 symbolisieren die durchgezogenen Pfeile die zeitliche Abfolge und die strichlierten Pfeile die indirekte Entscheidungsbeeinflussung der Spieler. 


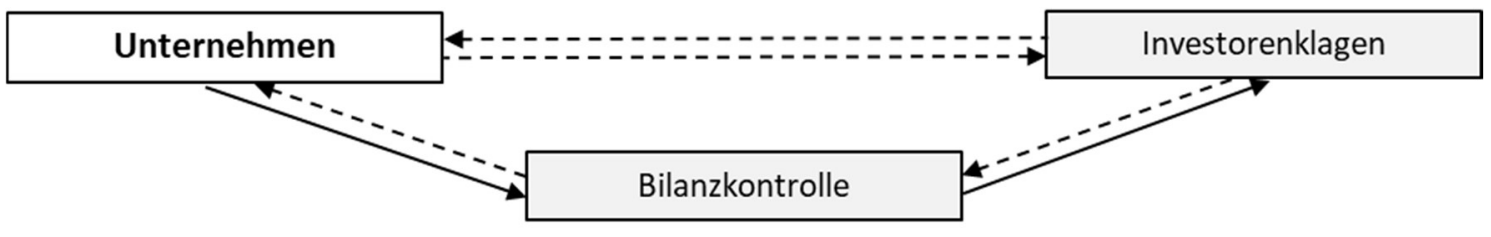

Abb. 3: Bilanzkontrolle und Investorenklagen.

Hier sind nun die Strategien aller drei Spieler von den Erwartungen über die der jeweils anderen abhängig, d.h. die Manipulation ist $m=m(\hat{c}, \hat{k})$, die Kontrollintensität ist $c=c(\hat{m}, \hat{k})$ und die Grenze, unter welcher geklagt wird, ist $k=k(\hat{m}, \hat{c})$. Wiederum erfolgt die Lösung durch ein Gleichgewicht in rationalen Erwartungen, was in diesem Fall recht komplexe Zusammenhänge ergibt.

Schantl und Wagenhofer (2020) zeigen folgende ökonomische Effekte einer Verstärkung der Bilanzkontrolle (konkret einer Vergrößerung der Stichprobe, ermöglicht z.B. durch ein größeres Budget):

- Investoren klagen weniger oft, d.h. nur für besonders ungünstige Signale, wenn die Bilanzkontrolle keinen Fehler veröffentlicht.

- Die Fehlerfeststellungsrate steigt, dies vor allem wegen der größeren Stichprobe.

- Die Kontrollintensität der Bilanzkontrolle sinkt, wenn das Klagerisiko relativ gering ist, und sie steigt, wenn dieses hoch ist.

- Das Unternehmen manipuliert weniger, wenn das Klagerisiko relativ gering ist, aber es manipuliert mehr, wenn dieses hoch ist.

Vor allem das letzte Resultat, dass es bei Verstärkung der Bilanzkontrolle zu mehrManipulation kommen kann, ist im ersten Moment überraschend, widerspricht es doch der Erwartung, dass mehr Kontrolle Manipulation verringern sollte. Die Intuition ist folgende: Eine höhere Fehlerfeststellungsrate durch die Bilanzkontrolle führt dazu, dass Investoren das Unternehmen ohne Risiko verklagen können. Umgekehrt, wird kein Fehler bekannt, ist es eher unwahrscheinlich, dass das Unternehmen manipuliert hat (sonst hätte die Bilanzkontrolle dies ja gefunden), und dies reduziert die Anreize zu klagen. Das führt zu einer Verdrängung der privaten Rechtsdurchsetzung durch die öffentliche Bilanzkontrolle. Je höher das zugrunde liegende Klagerisiko (z.B. wegen hoher Klagekosten für Investoren) ist, desto stärker ist dieser Verdrängungseffekt.

Aus Sicht des Unternehmens ist für die Manipulationsentscheidung relevant, wie hoch insgesamt die Wahrscheinlichkeit ist, dass dies eine Strafe nach sich zieht. Es spielt für den Abschreckungseffekt keine Rolle, wer - Bilanzkontrolle oder Gericht - die Manipulation herausfindet. Ist die private Rechtsdurchsetzung relativ schwach, also das Klagerisiko klein, ist der Substitutionseffekt aufgrund der Verstärkung der Bilanzkontrolle gering, und es ergibt sich in Summe eine größere Abschreckung von Manipulation. Umgekehrt, ist die private Rechtsdurchsetzung relativ stark, ist der Substitutionseffekt groß, und die Abschreckung sinkt insgesamt. Aus Sicht der Bilanzkontrolle bewirkt der Verdrängungseffekt von privater Rechtsdurchsetzung eine Reduktion der Kontrollanreize. Da aber die 
Manipulationswahrscheinlichkeit steigen kann, erhöht dies die Anreize. Welcher Effekt überwiegt, hängt wiederum vom Klagerisiko ab.

Schantl und Wagenhofer (2020) zeigen des Weiteren, dass solche gegenläufigen Ergebnisse - abhängig vom ursprünglich bestehenden Klagerisiko - auch für die Zahl der im Durchschnitt aufgedeckten Fehler und für das erwartete Klagerisiko (Summe der Klagen aufgrund von Fehlerfeststellung und sonstigen Klagen) insgesamt gelten. Insofern passt dies zur Auffassung der SEC, die 2018 argumentierte, dass die Anzahl der entdeckten Fehler und die Höhe der Strafzahlungen keine geeigneten Performancemaße sind. ${ }^{2}$

Insgesamt ist eine entscheidende Bedingung für eine Verhinderung von Manipulation durch Verstärkung der Bilanzkontrolle, dass die private Rechtsdurchsetzung als zweiter Kontrollmechanismus nicht besonders effektiv ist. Andernfalls kommt es zu starken Substitutionseffekten, die insgesamt eine Verschlechterung der Abschreckung von Manipulation bewirken können.

\section{$3 \quad$ Fazit}

Spieltheoretische Analysen von Kontrollmechanismen im Bereich der Finanzberichterstattung sind nützlich, um Interaktionen zwischen Kontrollmechanismen besser zu verstehen und ihre gemeinsame Wirkung auf Manipulationsverhinderung zu erkennen. Der wesentliche Schritt ist, Kontrollen nicht als "Maschine“ zu sehen, sondern als Institutionen, die strategische Entscheidungen treffen. Verändert man eine Größe, hat dies nicht nur Auswirkungen auf die direkt betroffene Kontrollinstanz, sondern auch auf alle anderen, die wiederum die Entscheidung der direkt betroffenen Instanz ändern, usw.

Ein besseres Verständnis möglicher Wirkungen von regulativen Maßnahmen ist Voraussetzung für eine gute Regulierung. Auf diese Weise wird ersichtlich, dass gut gemeinte und auf den ersten Blick plausible politische Maßnahmen auch nichtintendierte Wirkungen aufweisen und letztlich die Situation, die sie verbessern wollen, insgesamt sogar verschlechtern können. Intuitiv kann man sich Substitutionseffekte unter Kontrollmechanismen gut vorstellen, z.B. sinkt der Anreiz, selbst zu prüfen, wenn etwas ohnedies schon von einer oder gar mehreren anderen Instanzen geprüft wurde - man verlässt sich einfach gegenseitig aufeinander. In dem Artikel wurden nur zwei Kontrollmechanismen herausgehoben. Man kann sich aber gut vorstellen, dass nicht intendierte Wirkungen sogar noch häufiger auftreten, je mehr Kontrollinstanzen vorhanden sind. 


\section{Literaturverzeichnis}

Ewert, R., A. Wagenhofer. 2019. Effects of Increasing Enforcement on Financial Reporting Quality and Audit Quality. Journal of Accounting Research 57: 121-168. https://doi.org/10.1111/1475-679X.12251.

Hail, L., A. Tahoun, C. Wang. 2018. Corporate Scandals and Regulation. Journal of Accounting Research 56: 617-67. https://doi.org/10.1111/1475-679X.12201.

Michaels, D. 2018. SEC says don't judge its enforcement strength solely on volume of cases, fines. Wall Street Journal. [online]

https://www.wsj.com/articles/sec-says-dont-judge-enforcementstrength-solely-on-volume-fines-1537451398. [23.07.2021].

Schantl, S., A. Wagenhofer. 2020. Deterrence of Financial Misreporting when Public and Private Enforcement Strategically Interact. Journal of Accounting and Economics 70: 101311.

https://doi.org/10.1016/j.jacceco.2020.101311.

Quelle

Der Beitrag basiert auf "Effects of Increasing Enforcement on Financial Reporting Quality and Audit Quality" von Ralf Ewert und Alfred Wagenhofer erschienen in: Journal of Accounting Research", 57. Jg. 2019, S. 121-168, sowie "Deterrence of financial misreporting when public and private enforcement strategically interact" von Stefan F. Schantl und Alfred Wagenhofer erschienen in: Journal of Accounting and Economics, 70. Jg. 2020, Heft 1. 